\title{
Conocimiento y aplicación en prácticas higiénicas en la elaboración de alimentos y auto-reporte de intoxicaciones alimentarias en hogares chilenos
}

\author{
Jairo Torres ${ }^{1,2}$, Andres Voisier ${ }^{3}$ Ingrid Berríos ${ }^{3}$, Nicole Pitto y Samuel Durán Agüero 3
}

\section{Knowledge and application in hygienic practices in food preparation and self-report of food poisoning in Chilean homes}

Background: Foodborne diseases in the home are considered a problem at the national level, however, there is little information about household handling habits. Aim: To Identify the hygienic practices of people who prepare food at home and self-reporting food intoxication in Chilean homes. Material and Methods: A survey with 15 questions was made in google docs, containing questions about food handling practices and self-reporting food intoxication. Results: 2024 people were surveyed. Ninety six percent prepare food at home, of the total whom cook, $88 \%$ of respondents consider important good food handling practices at home, $76 \%$ say they know about the concept of cross contamination, however, more than $40 \%$ start their purchase in supermarkets with dairy and meat products which indicates that there is a high probability of cold chain loss, $56 \%$ of respondents indicate that they defrost food at room temperature, and with respect to fruits or vegetables only $12.5 \%$ use a disinfectant. Of the total number of respondents, $17 \%$ indicated that they had suffered food poisoning and of these only $64 \%$ attended the doctor. Conclusion: Although it is argued to have knowledge about good food handling practices, some important practices are not carried out.

Keywords: Hygienic practices; diseases transmitted by food; food handling.

Palabras clave: Prácticas higiénicas; enfermedades trasmitidas por alimentos; manipulación de alimentos.

\section{Introducción}

as enfermedades trasmitidas por alimentos (ETAs) generan una gran carga de enfermedades en todo el mundo; según la Organización Mundial de la Salud (OMS), en los países desarrollados se atribuye a éstas una gran pérdida de productividad y aumento en gastos de salud pública ${ }^{1}$. En cuanto a los países en vías de desarrollo, las ETAs son un foco importante de enfermedades y muerte, relacionándose directamente con un bajo nivel socio-económico, lo que genera un importante costo socio-económico ${ }^{1-3}$.

Las ETAs se manifiestan a través de un brote epidémico, al consumir alimentos que contengan microorganismos patógenos definiéndose un brote epidémico como la aparición de dos o más casos, con el consumo de un mismo alimento que produce un cuadro clínico ${ }^{1,4}$.

Las ETAs son originadas por alimentos contaminados principalmente por bacterias y virus, aunque también por hongos, parásitos, priones y toxinas ${ }^{3}$.

La OMS, informó que 70 a $80 \%$ de las enfermedades diarreicas agudas son ocasionadas por alimentos y agua contaminada $^{1}$. Se estima que la incidencia anual de dia- rreas en el concierto mundial, reportada por la OMS, es de 1.500 millones de $\operatorname{casos}^{2,4}$, considerando un porcentaje importante por la ingestión de productos alimentarios y agua contaminada ${ }^{1}$.

En Chile, durante el año 2016 se notificó un total de 1.096 brotes de ETAs. Desde Arica y Parinacota hasta Coquimbo se han presentado, de forma mantenida en el tiempo, las mayores tasas de notificación por casos asociados de brotes por ETAs. La Región de Tarapacá tuvo la tasa más alta con 119,2 casos por 100.000 habts., seguida de Arica y Parinacota 115, 2 por 100.000 habts., mientras que las tasas más bajas se presentan en la Región Metropolitana y Región de Los Ríos con 17,0 y 16,7 casos por 100.000 habts., respectivamente 5 .

El análisis de los brotes de ETA, concluye que fueron debidos en $43,5 \%$ a las comidas y platos preparados (con diversidad de ingredientes), en $28,9 \%$ a pescados y productos de pesca, en 7,2\% a las carnes y productos cárnicos y $6,4 \%$ fueron debidos a huevos. Además, se observa que el principal lugar donde ocurrieron las intoxicaciones fue en el hogar: $(57,1 \%)$, en segundo lugar, en restaurantes $(18,7 \%)$ y tercer lugar $(9,6 \%)$ en los casinos, clubes sociales y cocinerías ${ }^{5}$.
Universidad Tecnológica de Chile INACAP. Chile.

${ }^{2}$ Facultad de Ciencias de la Salud, Universidad Autónoma de Chile. Chile.

${ }^{3}$ Escuela de Nutrición y Dietética. Facultad de Ciencias para el Cuidado de la Salud, Universidad San Sebastián. Chile.

Todos los autores declaran no poseer conflicto de intereses.

Proyecto autofinanciado.

Recibido: 1 de febrero de 2018 Aceptado: 16 de julio de 2018

Correspondencia a: Jairo Alonso Torres jairoalonsot@gmail.com 
Los principales diagnósticos clínicos, se asociaron a Salmonella spp y envenenamiento escombroide (histamínico) por pescado, con 84 y 81 brotes, respectivamente.

En el 2016, la Región Metropolitana presentó la mayor concentración de ETAs, con 106 brotes $(39,6 \%)$ en el hogar, siendo el lugar más frecuente de la pérdida de la inocuidad alimentaria. Por lo tanto, el hogar es el lugar de mayor riesgo epidemiológico para contraer enfermedades entéricas ${ }^{6}$.

Los brotes de ETAs en los hogares se generan a causa de las inadecuadas prácticas de higiene, tanto, en la manipulación como en el mantenimiento de los alimentos en el hogar, entre las que se destaca: una refrigeración inadecuada, un lapso de tiempo superior a $12 \mathrm{~h}$, o incluso más, entre la preparación del alimento y el consumo, personas en el hogar que padezcan infecciones y manipulan alimentos, recalentamiento inadecuado, mantenimiento de los alimentos que están preparados en lugares no aptos y/o a temperaturas que favorecen el crecimiento microbiano, consumir alimentos crudos de alto riesgo o que contengan algún ingrediente contaminado, limpieza no realizada de forma correcta de los utensilios y equipos de cocina en el hogar (refrigerador, cocina, microondas, hervidor, entre otros $)^{7-9}$.

En la población chilena existen inadecuadas prácticas higiénicas, tanto en lugares públicos de alimentación como en los hogares ${ }^{2}$; por el contrario, en otros países se argumenta que existe un conocimiento adecuado ${ }^{10}$.

Para evitar los brotes de ETAs, hay que considerar una correcta mantención de la cadena de frío, cocción adecuada de los alimentos y evitar la contaminación cruzada $^{11,12}$. En la cadena de producción, desde la fabricación hasta el consumo final, comparten la responsabilidad de la inocuidad alimentaria, según lo estipula el Codex Alimentarius de la $\mathrm{FAO}^{2}$.

El objetivo de este estudio es determinar el conocimiento y aplicación en prácticas higiénicas en la elaboración de alimentos y auto-reporte de intoxicaciones alimentarias en hogares chilenos.

\section{Material y Métodos}

Estudio descriptivo, transversal, para lo cual se elaboró una encuesta online utilizando google drive. La encuesta se realizó en el período de abril a mayo 2017. Para la obtención de la información se llevaron a cabo las siguientes etapas:

\section{Elaboración de la encuesta}

Se diseñó una encuesta corta en formato google drive. Fue validada por método Delphi por expertos en el área (docentes universitarios y expertos en inocuidad que trabajan en la industria de alimentos), y autorizada por el Comité de Ética de la Universidad San Sebastián, Chile. La encuesta consta de consentimiento informado y un total de 15 preguntas; algunas de caracterización general (sexo, edad), preguntas con respuesta dicotómica (si o no) sobre importancia de la manipulación de alimentos a nivel hogareño, dónde compra y en qué orden compra y almacena los alimentos (lácteos, carnes y vegetales). También se les preguntó por el manejo de algunas prácticas básicas de manipulación de alimentos, tales como: lavado, desinfección, contaminación cruzada y cadena de frío y si habían experimentado algún tipo de intoxicación alimentaria por comida elaborada en el hogar y qué medidas habían tomado frente a una intoxicación (automedicación, asistencia a médico u otros).

\section{Aplicación de la encuesta}

El estudio se efectuó en Chile, con diversos colaboradores que se encargaron de realizar la difusión de la encuesta por redes sociales como Facebook, Twitter, WhatsApp, mail, bases de datos, entre otras. El criterio de inclusión para el presente estudio era ser mayor de 18 años, chileno y que preparara alimentos en su hogar. Criterio de exclusión fue ser persona no vidente.

\section{Análisis estadístico}

Los resultados se presentan como porcentajes según el tipo de variable. Los datos fueron recopilados en una plantilla EXCEL, las variables cualitativas se analizaron con la prueba de $\chi^{2}$, los datos fueron procesados con el paquete estadístico SPSS $22.0 \AA$.

\section{Resultados}

Del total de los 2.024 encuestados, $82,7 \%$ es de sexo femenino.

Con respecto al grupo etario, quienes más respondieron la encuesta son personas entre 18 y 30 años (46,2\%), seguido del grupo de 31 a 40 años $(27,3 \%)$, el grupo de 41 a 50 años (13,4\%), y finalmente los grupos de 51 a 60 y 60 años o más $(9,1$ y 3,9\%, respectivamente).

Con respecto al nivel educacional, 1,6\% tiene enseñanza básica completa, 22,3\% enseñanza media completa, $15,2 \%$ técnico superior completa, $45,5 \%$ universitaria completa y $15,4 \%$ tiene un postgrado.

Noventa y seis por ciento elabora alimentos en el hogar; de ellos, $88 \%$ considera importante las buenas prácticas de manipulación, 11\% medianamente importante y sólo 1\% no las considera importantes (Figura 1).

En relación del lugar de compra los abarrotes, conservas, lácteos, huevos, carnes y derivados prefieren comprarlos en supermercados, mientras que adquirir las frutas y verduras en ferias libres. 


\begin{tabular}{|c|c|c|c|c|c|}
\hline Edad (años) & $18-30$ & $31-40$ & $41-50$ & $51-60$ & $>60$ años \\
\hline Número/(\%) & 935 / (46,2\%) & $553 /(27,2 \%)$ & $272 /(13,4 \%)$ & 185 / (9,1\%) & 79 / (3,9\%) \\
\hline \multicolumn{6}{|l|}{ Sexo } \\
\hline Mujeres & $1.673(82,7 \%)$ & & & & \\
\hline Hombres & $351(17,3 \%)$ & & & & \\
\hline
\end{tabular}

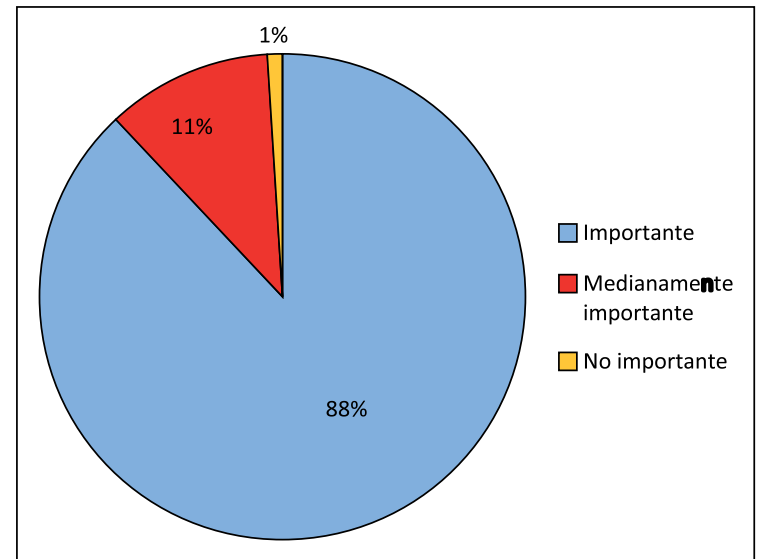

Figura 1. Importancia sobre la correcta manipulación de los alimentos en el hogar.

Con respecto al orden en que efectúa la compra, $43,4 \%$ adquiere los lácteos al principio o intermedio y 52,5\% lo hace así con carnes y derivados (Figura 2).

Al preguntar por las prácticas de almacenamiento en el hogar, 21,8\% de los encuestados no aplica criterio alguno. Los encuestados indican que, al momento de preparar alguna fruta o verdura, 86,3\% la lava bajo el agua, 12,5\% la lava usando desinfectante y 1,2\% las prepara sin lavar.

Se cuantificó que $56,5 \%$ de los encuestados descongela los alimentos a temperatura ambiente y $64,1 \%$ no almacena de inmediato la comida (Figura 3). En cambio, $97,9 \%$ se lava las manos, previo a la manipulación y $94,3 \%$ utiliza recipientes tapados.

Setenta y seis por ciento de los encuestados aseguran saber sobre el concepto de contaminación cruzada y manifiestan ejecutar correctamente algunas prácticas de manipulación de alimentos a excepción de la forma de descongelación de los productos; $56 \%$ indica que descongelan a temperatura ambiente.

Finalmente, del total de encuestados, 17\% indica haber experimentado una intoxicación alimentaria y de éstas, 64\% asiste al médico, el porcentaje restante se auto-medica (Figura 4 y 5).

En la Tabla 2 se presenta la comparación de conoci-

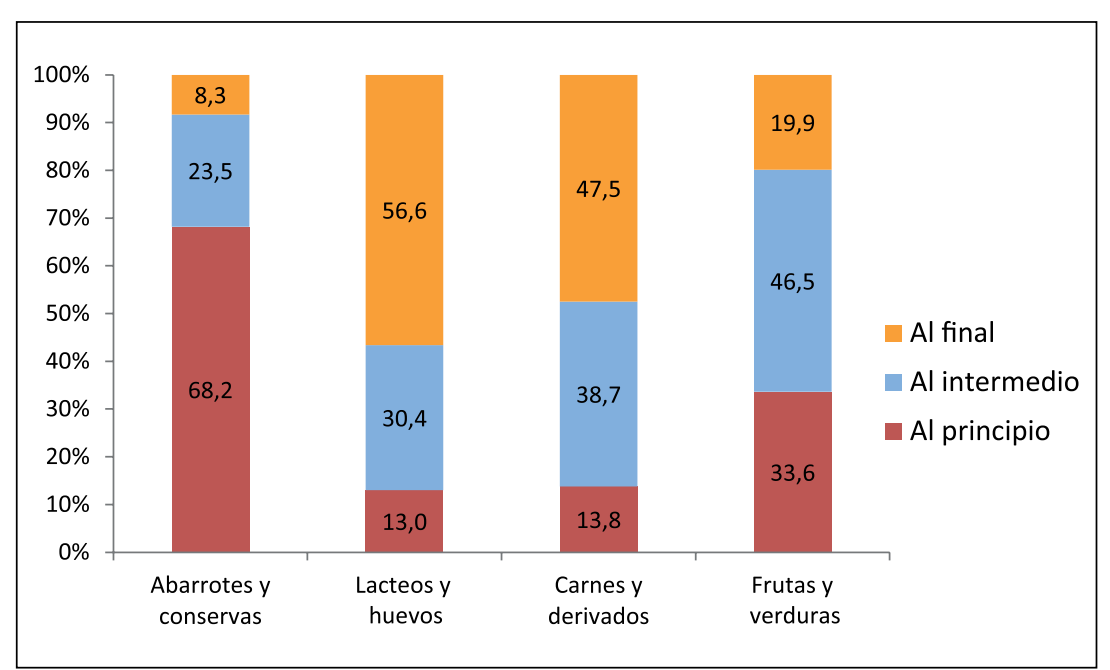

Figura 2. Selección en el orden de compra de alimentos en el supermercado.

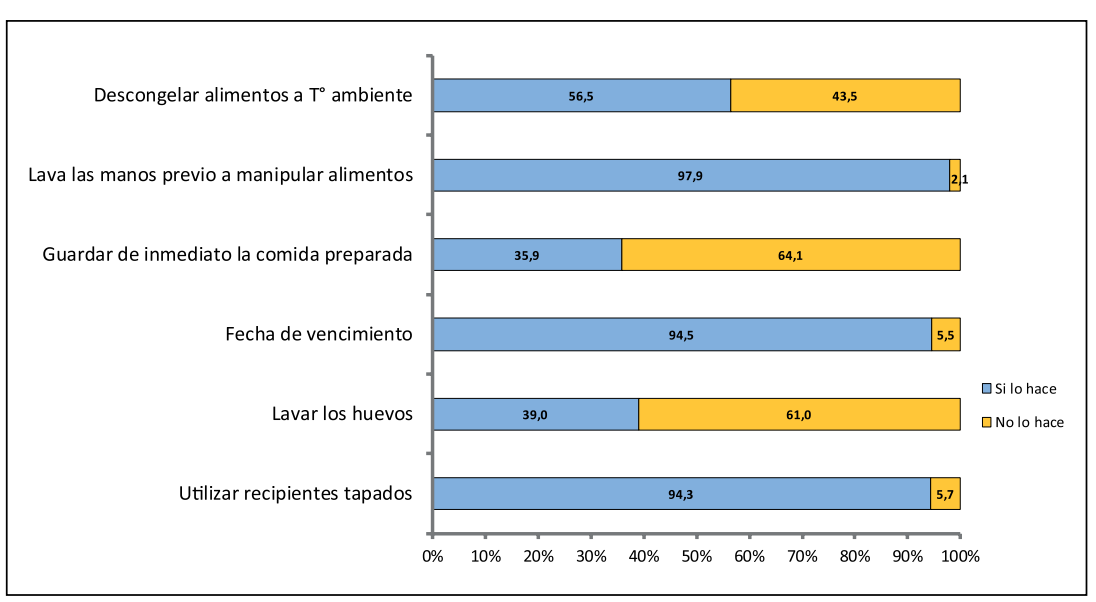

Figura 3. Prácticas de higiene en la elaboración de los alimentos en el hogar.

mientos y prácticas higiénicas según nivel de estudios. Se observa que, en general, no se presentan diferencias, excepto en utilizar, en menor porcentaje, los recipientes con tapa y sellados y seguir un orden de almacenamiento 


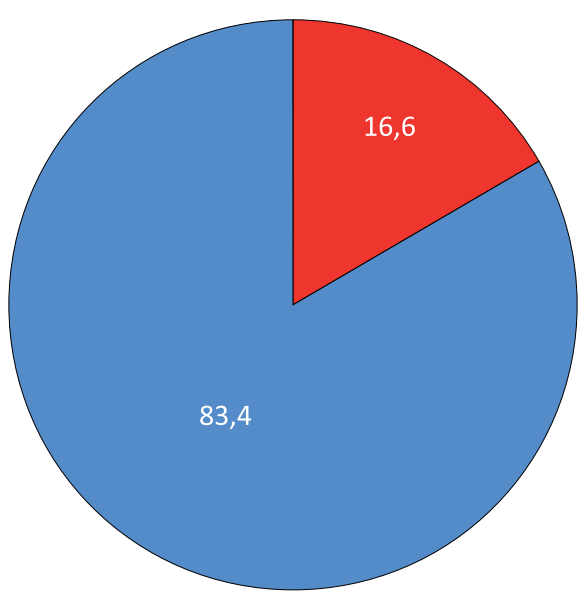

Si han sufrido intoxicación No han sufrido intoxicación

Figura 4. Autorreporte (\%) de intoxicaciones alimentarias por alimentos elaborados en el hogar.

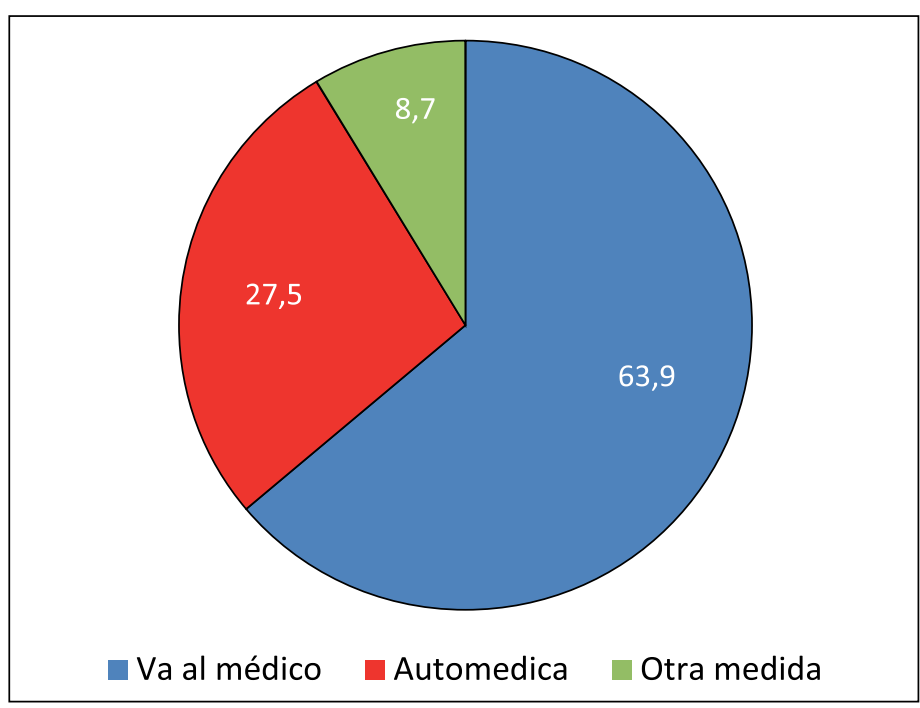

Figura 5. Medidas a tomar frente a una intoxicación alimentaria.

\begin{tabular}{|c|c|c|c|c|c|}
\hline & & Básica & Media & Superior & Valor $p$ \\
\hline \multirow{3}{*}{$\begin{array}{l}\text { ¿Cuán importante considera usted la manipulación correcta de } \\
\text { alimentos en el hogar, tomando en cuenta temperaturas de } \\
\text { almacenamiento, procedimientos de lavado, contaminación, etc? }\end{array}$} & Poco (\%) & 3,2 & 1,0 & 0,8 & \multirow[t]{3}{*}{0,183} \\
\hline & Medio (\%) & 6,5 & 13,8 & 10,7 & \\
\hline & Importante (\%) & 90,3 & 85,2 & 88,5 & \\
\hline \multirow{2}{*}{$\begin{array}{l}\text { Al llegar a su casa y guardar los alimentos en el refrigerador, usted } \\
\text { almacena }\end{array}$} & Con orden establecido (\%) & 83,9 & 68,2 & 80,9 & \multirow[t]{2}{*}{0,001} \\
\hline & Sin orden (\%) & 16,1 & 31,8 & 19,1 & \\
\hline \multirow[t]{3}{*}{ Al momento de preparar alguna fruta o verdura, usted } & Sin lavar ni desinfectar (\%) & 0,0 & 1,2 & 1,2 & \multirow[t]{3}{*}{0,881} \\
\hline & Solo lavado (\%) & 89,2 & 87,2 & 86,0 & \\
\hline & Lavado y desinfectado (\%) & 10,8 & 11,6 & 12,8 & \\
\hline \multirow{2}{*}{$\begin{array}{l}\text { Utiliza recipientes tapados o sellados para guardar los alimentos } \\
\text { ya preparados }\end{array}$} & No (\%) & 6,5 & 9,0 & 4,7 & \multirow[t]{2}{*}{0,004} \\
\hline & Sí (\%) & 93,5 & 91,0 & 95,3 & \\
\hline \multirow[t]{2}{*}{ Revisa fechas de vencimiento } & No (\%) & 13,0 & 6,4 & 5,1 & \multirow[t]{2}{*}{0,117} \\
\hline & Sí (\%) & 87,0 & 93,6 & 94,9 & \\
\hline \multirow[t]{2}{*}{ Al cocinar, guarda en el refrigerador la comida preparada } & No (\%) & 67,7 & 68,1 & 62,9 & \multirow[t]{2}{*}{0,120} \\
\hline & Sí (\%) & 32,3 & 31,8 & 37,1 & \\
\hline \multirow[t]{2}{*}{ Lava las manos antes de manipular alimentos } & No (\%) & 3,2 & 22,1 & 2,1 & \multirow[t]{2}{*}{0,910} \\
\hline & Sí (\%) & 96,8 & 97,9 & 97,9 & \\
\hline \multirow[t]{2}{*}{ Descongela los alimentos a temperatura ambiente } & No (\%) & 77,5 & 66,1 & 53,4 & \multirow[t]{2}{*}{0,001} \\
\hline & Sí (\%) & 22,5 & 33,9 & 46,6 & \\
\hline \multirow[t]{2}{*}{ Usted sabe en qué consiste la contaminación cruzada } & No $(\%)$ & 71,0 & 37,6 & 19,6 & \multirow[t]{2}{*}{0,001} \\
\hline & Sí (\%) & 29,0 & 62,4 & 80,4 & \\
\hline
\end{tabular}


en el refrigerador, donde los sujetos de escolaridad media presentan el mayor porcentaje de personas que guardan sin orden alguno. Con respecto a descongelar a temperatura ambiente, esta conducta es más frecuente en los sujetos de mayor escolaridad, situación contraria ocurre con el conocimiento sobre contaminación cruzada.

\section{Discusión}

El principal resultado del presente estudio es que, a pesar de que se considera importante las buenas prácticas de manipulación de alimentos en el hogar, estas prácticas no son ejecutadas.

Es relevante considerar que para asegurar la inocuidad alimentaria se deben controlar las condiciones básicas tales como compra, manipulación y almacenamiento de alimentos, es decir, buenas prácticas higiénicas y/o de manipulación. Se destaca la preocupación por la inocuidad que deben presentar los alimentos industrializados, pero también se debe poner énfasis en los alimentos que se preparan en el hogar, ya que toda manipulación que reciben los alimentos puede poner en riesgo la salud del ser humano ${ }^{13}$.

Según los encuestados, el lugar más frecuente para adquirir frutas y verduras es las ferias libres, posiblemente porque existe una mayor variedad y frescura de estos productos; para los otros alimentos, las compras se realizan en supermercado y almacenes, posiblemente por una mayor accesibilidad.

Es importante saber que se debe garantizar la inocuidad en todo el proceso productivo, es decir, desde el campo a la mesa. Para ello, se requiere de las buenas prácticas de manufactura que garantizan la calidad, seguridad e inocuidad de los alimentos, las que se encargan de establecer métodos hacia una producción, manipulación, transporte, almacenamiento y/o distribución correcta,

Para garantizar una correcta manipulación de alimentos en el hogar, se debe considerar desde la elección, compra, transporte, almacenamiento, descongelación (cuando corresponda), preparación y mantención.

En la etapa de compra de los productos lácteos y cárnicos, sobre $40 \%$ es realizado al inicio o intermedio de esta, lo que indica que existe una alta probabilidad de pérdida de cadena de frio $\mathrm{y}$, por consiguiente, un factor de riesgo.

Para prevenir las ETAs, se requiere el control de factores como la calidad de los ingredientes, las condiciones ambientales, la forma de compra, transporte y almacenamiento y el correcto uso y mantenimiento de utensilios y equipos y de las técnicas de higiene apropiadas de preparación $^{12,14}$.

Nuestros resultados muestran que un gran porcentaje de la población identifica el concepto de contaminación cruzada y algunas prácticas básicas de manipulación de alimentos; no obstante, $56 \%$ descongela los alimentos a temperatura ambiente, que es una práctica inadecuada porque propicia la proliferación de microrganismos ${ }^{15}$.

La correcta manipulación en la etapa de almacenamiento, elaboración, mantención y consumo de los alimentos en el hogar es de vital importancia para prevenir las enfermedades de transmisión por alimentos. El presente estudio refleja que los participantes tienen el conocimiento y a la vez saben que es importante llevarlas a cabo, pero existe una escasa aplicación, excepto el lavado de manos, verificación de fecha de vencimiento y uso de recipientes tapados para la mantención.

El Ministerio de Salud (MINSAL) recomienda las siguientes medidas de prevención: Lavar las manos con agua y jabón cuando se va a manipular y preparar los alimentos, antes de comer y después de ir al baño o cambiar pañales, beber siempre agua potable o hervida la cual se deja enfriar y se almacena, limpiar y desinfectar mesones donde se preparan alimentos (especialmente aquellos en contacto con alimentos crudos), manipular en forma separada carnes y mariscos crudos y, de la misma forma, aquellos vegetales que crecen al ras del suelo del resto de los alimentos, separar los alimentos crudos de los cocinados o preparados listos para su consumo, consumir carnes, pescados y mariscos bien cocidos, mantener los alimentos a una temperatura adecuada o segura, tanto para mantener la cadena de frio o caliente, lavar todas las verduras y frutas, incluso las pre-elaboradas, no consumir mayonesa casera y comprar o consumir alimentos en lugares autorizados ${ }^{16,17}$. Se recomienda: consumir sólo productos lácteos y sus derivados pasteurizados (leche, queso gauda, queso fresco, quesillo, chanco, mantecoso, entre otros), mantener estos productos y las cecinas refrigeradas, recordar que calentar alimentos en el microondas no elimina las bacterias. No consumir: pescados y carnes crudas o parcialmente cocidas (vacuno, cordero, cerdo, aves, sushi, ceviche, carpaccio, pescados ahumados, entre otros), salchichas crudas o mal cocinadas, paté, pastas de queso o jamón de expendio a granel, alimentos de origen desconocido o de comercio informal o no autorizado ${ }^{18}$.

Un estudio realizado en Colombia encontró que existía una relación entre inseguridad alimentaria y bajo nivel de ingresos ${ }^{18}$; sin embargo, para el caso de Chile pudimos detectar que las prácticas inadecuadas de manipulación de alimentos se dan en todos los niveles educacionales. Otro estudio, realizado en Nigeria, comprobó que el riesgo de contaminación y proliferación de microrganismos aumentó al almacenar los alimentos a temperatura ambiente, temperaturas insuficientemente altas para recalentar los alimentos y añadiendo ingredientes contaminados ${ }^{19}$. Un tercer estudio, realizado en Estados Unidos de América, 
mostró, en cambio, que la contaminación de una esponja o paño de cocina con coliformes fecales o $S$. aureus fue predictora de otras superficies en la cocina que tienen la misma contaminación, lo que indica que las esponjas y los paños de cocina son tanto reservorios como vectores de bacterias en la cocina ${ }^{20}$.

Diez y siete por ciento de los encuestados indica haber experimentado una intoxicación por alimentos preparados en el hogar, tasa considerada relativamente alta. Lo preocupante de esta situación es que $34 \%$ de las personas que se intoxican refiere no asistir al médico, sino que se auto-medican, casos que no quedan reportados en los sistemas de salud, produciendo un subregistro. Lo recomendable, frente a una intoxicación alimentaria es asistir al médico ${ }^{21}$ y por el contrario, no auto-medicarse, ya que puede estar expuestos ante un microorganismo potencialmente patógeno y auto medicarse sin prescripción médica puede prolongar la diarrea y el estado de intoxicación de la persona ${ }^{21,22}$.

En Chile, se encargan de recibir las notificaciones de los brotes de las enfermedades transmitidas por alimentos (ETAs) las Secretarías Regionales Ministeriales de Salud (SEREMI), quienes tienen la responsabilidad de inspeccionar y notificar las Enfermedades Transmisibles de Declaración Obligatoria, y así llevar a cabo la confirmación diagnóstica de cada uno de los casos causados por las ETAs ${ }^{23}$. Sin embargo, en nuestro y en otros países no se puede dimensionar la prevalencia de agentes virales, debido a los escasos estudios de laboratorio, que puedan avalar el tipo de agente en los brotes de sospecha ${ }^{22,4}$.

Las ETAs producidas por alimentos en el hogar son uno de los problemas a nivel global; por ende, se requiere intervenir con acciones de promoción y prevención, resultando necesario mantener una estricta vigilancia epidemiológica de los alimentos, para poder aplicar de forma oportuna medidas preventivas y correctoras ${ }^{4}$.

En algunos países se han usados estrategias educativas en niños para que desde la infancia reconozcan las buenas prácticas de manipulación de alimentos ${ }^{24}$. Es importante ampliar diversos canales de comunicación para difundir las practicas higiénicas de manipulación de alimentos ${ }^{25,26}$.

Es importante mencionar que el presente es el primer estudio nacional con un número de encuestados representativo. La principal limitación del estudio es que quedan excluidas las personas que no tienen acceso a redes sociales, es decir población de menos recursos económicos y personas mayores.

\section{Conclusión}

En el presente estudio de investigación hay un porcentaje considerable que cree importante utilizar las prácticas de higiene en la manipulación de los alimentos en el hogar.

Las personas al comprar en los supermercados realizan una selección de orden de compra inadecuada, lo que genera pérdida en la cadena frío de los productos.

La mayoría de los casos tienen malas prácticas de higiene, excepto lavarse las manos, previo a manipular los alimentos, ver la fecha de vencimiento del mismo y usar recipientes tapados.

Ochenta y tres por ciento de los participantes no ha experimentado una intoxicación alimentaria. Y el 17\% restante que sí la ha sufrido de forma prioritaria van al médico, luego se auto medican y un porcentaje pequeño toma otras medidas.

Se hace necesario aumentar la educación en practicas de higiene en la población general.

\section{Resumen}

Introducción: Las enfermedades transmitidas por alimentos en el hogar son consideradas una problemática nacional; sin embargo, existe poca información sobre hábitos de manipulación en el hogar. Objetivo: Determinar el conocimiento y aplicación de prácticas higiénicas en la elaboración de alimentos y auto-reporte de intoxicaciones alimentarias en hogares chilenos. Material y Métodos: Se confeccionó una encuesta de 15 preguntas en formato google docs, se consultó sobre prácticas de manipulación de alimentos en el hogar y auto-reporte de intoxicaciones alimentarias. Resultados: Se encuestó a 2.024 personas. Noventa y seis por ciento elabora alimentos en el hogar; del total que lo hace, $88 \%$ de los encuestados considera importante las buenas prácticas de manipulación de alimentos en el hogar y $76 \%$ asegura saber sobre la contaminación cruzada. Más de $40 \%$ inicia su compra en los supermercados con productos lácteos y cárnicos, generando una pérdida de cadena de frío; $56 \%$ de los encuestados indica que descongelan los alimentos a temperatura ambiente. Con respecto a frutas o verduras, sólo $12,5 \%$ utiliza un desinfectante. Del total de los que elaboran alimentos en el hogar, $17 \%$ indica haber sufrido una intoxicación alimentaria y de éstas, sólo $64 \%$ asiste al médico. Conclusión: A pesar de que se considera importante las buenas prácticas de manipulación de alimentos en el hogar, estas prácticas no son ejecutadas. 


\section{Referencias bibliográficas}

1.- Kirk M, Pires S, Black R, Caipo M, Crump J, Devleesschauwer B, et al. World Health Organization estimates of the global and regional disease burden of 22 foodborne bacterial, protozoal, and viral diseases 2010: A data synthesis. PLoS Med 2015; 12 (12): e1001940. doi: 10.1371/journal.pmed.1001921.

2.- Guo S, Lin D, Wang L L, Hu H. Monitoring the results of foodborne diseases in sentinel hospitals in Wenzhou City, China from 2014 to 2015. Iran J Public Health 2018; 47 (5): 67481. https://www.ncbi.nlm.nih.gov/pmc/articles/ PMC6005988/pdf/IJPH-47-674.pdf

3.- López D, Rivero Á, Martínez T, Alegret R. Enfermedades transmitidas por alimentos en Villa Clara. Rev Cuba Hig Epidemiol. 2013; 51 (2): 203-13. http://scielo.sld.cu/pdf/hie/v51n2/ hie09213.pdf

4.- Rumble C, Addiman S, Balasegaram S, Chima K, Ready D, Heard J, et al. Role of food handlers in norovirus outbreaks in London and South East England, 2013 to 2015. J Food Prot. 2017; 80 (2): 257-64. doi: 10.4315/0362-028X. JFP-16-083.

5.- Departamento de Epidemiología Ministerio de Salud Chile. Boletín Epidemiológico Trimestral, Volumen 112, $N^{\circ} 3$, año 2016 [Internet]. [citado el 1 de mayo de 2017]. Disponible en: http://epi.minsal.cl/wp content/ uploads/2016/11/BET_ETA_ENERO_ SEPTIEMBRE_2016.pdf.

6.- SEREMI Región Metropolitana, Ministerio de salud, Situación epidemiológica de los Brotes de Enfermedad Transmitida por Alimentos (ETA). Región Metropolitana. Año 2016. Resultados de la vigilancia. [Internet]. [citado el 18 de mayo de 2017]. Disponible en: http:// seremi13.redsalud.gob.cl/wrdprss_minsal/ wp-content/uploads/2016/02/Informe-anualETA-2016.pdf.

7.- Whiley H, Clarke B, Ross K. Knowledge and attitudes towards handling eggs in the home: an unexplored food safety issue? Int J Environ Res Public Health. 2017;14 (1). pii: E48. doi: 10.3390/ijerph14010048.

8.- Rossi M, Stedefeldt E, Da Cunha D, Rosso V. Food safety knowledge, optimistic bias and risk perception among food handlers in institutional food services. Food Control, 2017; 73: 681-8. DOI: 10.1016/j.foodcont.20.
9.- Kendall P, Scharff R, Baker S, LeJeune J, Sofos J, Medeiros L. Food safety instruction improves knowledge and behavior risk and protection factors for foodborne illnesses in pregnant populations. Matern Child Health J 2017; 21 (8): 1686-98. doi: 10.1007/s10995-017-2291-2.

10.- Akabanda F, Hlortsi E H, Owusu-Kwarteng J. Food safety knowledge, attitudes and practices of institutional food-handlers in Ghana. BMC Public Health 2017; 17 (1): 40. doi: 10.1186/ s12889-016-3986-9.

11.- Assunta B, Souza G, Teo A, Paz C. Pozzagnol M. Condiciones socioeconómicas e higiénicosanitarias como dimensiones de la seguridad alimentaria y nutricional. Rev Chil Nutr 2016; 43 (1): 62-7. http://dx.doi.org/10.4067/S071775182016000100009.

12.- Jung J, Friedrich L, Danyluk, M, Schaffner D. Quantification of transfer of Salmonella from citrus fruits to peel, edible portion, and gloved hands during hand peeling. J Food Prot 2017; 80 (6): 933-9. doi: 10.4315/0362-028X.JFP-16423.

13.- Assunta B, Souza L, Arruda C, Pozzagnol M. Condiciones socioeconómicas e higiénicosanitarias como dimensiones de la seguridad alimentaria y nutricional. Rev Chil Nutr. 2016; 43 (1): 62-7. http://dx.doi.org/10.4067/S071775182016000100009 .

14.- Makwana P, Nayak J, Brahmbhatt M, Chaudhary J. Detection of Salmonella spp. from chevon, mutton and its environment in retail meat shops in Anand city (Gujarat), India. Vet World. 2015; 8(3): 388-92. doi: 10.14202/ vetworld.2015.388-392.

15.- Código de prácticas de higiene para los alimentos precocinados y cocinados utilizados en los servicios de comidas para colectividades www.fao.org/input/download/standards/25/ CXP_039s.pdf.

16.- SEREMI de Salud R. M. Situación epidemiológica de los Brotes de Enfermedad Transmitida por Alimentos (ETA). Región Metropolitana. Año 2016. Resultados de La Vigilancia. [Internet]. [citado el 31 de enero de 2018]. Disponible en: http://seremi13. redsalud.gob.cl/wrdprss_minsal/wp-content/ uploads/2016/02/Informe-anual-ETA-2016.pdf.

17.- Lozano G, Estrada A. Seguridad alimentaria en hogares de Acandí, Darién caribe colombiano: el aporte del caracol Cittarium pica "la cigua". Rev Chil Nutr 2008; 35(4): 460-70.
18.- Mejía J. Mejores prácticas en preparación de alimentos en micro y pequeña empresa. Entorno 2015; 0 (56): 16-21.

19.- Ehiri J E, Azubuike M C, Ubbaonu C N, Anyanwu E C, Ibe KM, Ogbonna MO. Critical control points of complementary food preparation and handling in Eastern Nigeria. Bull World Health Organ. 2001; 79(5): 423-33. https://www.ncbi.nlm.nih.gov/pmc/articles/ PMC2566429/

20.- Borrusso P A, Quinlan J J. Prevalence of pathogens and indicator organisms in home kitchens and correlation with unsafe food handling practices and conditions. $\mathrm{J}$ Food Prot 2017; 80 (4): 590-7. https://doi. org/10.4315/0362-028X.JFP-16-354.

21.- Ruiz Á, Pérez A. Automedicación y términos relacionados: una reflexión conceptual. Rev Cienc Salud 2011; 9 (1): 83-97. https://revistas. urosario.edu.co/index.php/revsalud/article/ viewFile/1551/1382

22.- Miranda S, Emilio L. Automedicación en locales de farmacia de la Región Metropolitana. Universidad Andres Bello, Escuela de Química y Farmacia. 2008 [citado el 30 de enero de 2018]; Disponible en: http://repositorio.unab.cl/xmlui/ bitstream/handle/ria/1789/sepulveda le.pdf? sequence $=1$ \&isAllowed $=y$

23.- Circular de investigación epidemiológica y control ambiental de brotes de enfermedades transmitidas por los alimentos. http:// info.seremisaludatacama.cl/documents/ epidemiologia/Normas/Circulares $\% 20 \mathrm{y} \% 20$ Normas/ETA/Circular-Brote-ETA-Copiaautorizada.pdf.

24.- Traversa A, Adriano D, Bellio A, Bianchi D, Gallina S, Ippolito C, et al. Food safety and sustainable nutrition workshops: educational experiences for primary school children in Turin, Italy. Ital J Food Saf 2017; 6 (1): 61-77.doi: 10.4081/ ijfs.2017.6177

25.- Huang R, Wei X, Pang J, Sun Y. Analysis on the choice of food safety information acquisition channel. J Food Saf Quality 2017; 8 (2): 698-703.

26.- Kang H J, Lee M W, Hwang I K, Kim J W. Development of safe food handling guidelines for Korean consumers. J Food Prot 2015; 78 (8): 1541-6. doi: 10.4315/0362-028X.JFP-14506 . 